\title{
Conductive bacterial cellulose-polyaniline blends: Influence of the matrix and synthesis conditions
}

\author{
Emanuel Alonso $^{\mathrm{a}}$, Marisa Faria ${ }^{\mathrm{a}}$, Faranak Mohammadkazemi ${ }^{\mathrm{b}}$, Matic Resnik ${ }^{\mathrm{c}}$, Artur Ferreira $^{\mathrm{d}}$, \\ Nereida Cordeiro ${ }^{\mathrm{a}, *}$

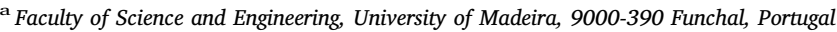 \\ ${ }^{\mathrm{b}}$ Department of Biosystems, Faculty of New Technologies Engineering, Shadid Beheshti University, Science and Research Campus, Zirab, Savadkooh, Mazandaram, Iran \\ c Jožef Stefan Institute, Jamova 39, SI-1000 Ljubljana, Slovenia \\ ${ }^{\mathrm{d}}$ CICECO - Aveiro Institute of Materials, Águeda School of Technology and Management, University of Aveiro, 3810-193 Aveiro, Portugal
}

\section{A R T I C L E I N F O}

\section{Keywords:}

Bacterial cellulose

Polyaniline

Blend membrane

Inverse gas chromatography

\begin{abstract}
A B S T R A C T
Bacterial cellulose/polyaniline (BC/PANi) blends present a great potential for several applications. The current study evaluates the impact of using different BC matrixes (drained, freeze-dried and regenerated) and different synthesis conditions (in situ and ex situ) to improve the inherent properties of BC, which were monitored through FTIR-ATR, EDX, XRD, SEM, AFM, swelling, contact angle measurement and IGC. The employment of in situ polymerization onto drained $\mathrm{BC}$ presented the most conductive membrane $\left(1.4 \times 10^{-1} \mathrm{~S} / \mathrm{cm}\right)$. The crystallinity, swelling capacity, surface energy and acid/base behavior of the BC membranes is substantially modified upon PANi incorporation, being dependent on the BC matrix used, being the freeze-dried BC blends the ones with highest crystallinity (up to $54 \%$ ), swelling capacity (up to $414 \%$ ) and surface energy (up to $75.0 \mathrm{~mJ} / \mathrm{m}^{2}$ ). Hence, this work evidenced that the final properties of the BC/PANi blends are greatly influenced by both the BC matrixes and synthesis methods employed.
\end{abstract}

\section{Introduction}

Intrinsically conductive polymers (ICP) are a special type of polymers that are characterized by a conjugated $\pi$-electron backbone which confers the unusual property to conduct electricity (Catedral, Tapia, \& Sarmago, 2004). Among the different ICP, polyaniline (PANi) is well known for its environmental stability as well as ease of synthesis. This way, it presents a great potential to be applied as thin film transistors, supercapacitors, engineering scaffolds, implantable biosensors and implantable neural prosthetic devices (Kaur, Adhikari, Cass, Bown, \& Gunatillake, 2015; Sapurina \& Shishov, 2012). Although, the application of this polymer is restricted due to its poor mechanical properties. This way, in the recent years, considerable work has been done regarding ways to improve the processing properties of PANi through the production of blends with natural polymers. Amongst the different matrixes used on PANi synthesis, natural fibers such as bacterial cellulose presented to be promising since the current environmental issues shows a pressing need for sustainable, degradable and recyclable materials while performing at the same level as non-degradable materials.

Bacterial cellulose (BC) is the purest form of cellulose found in nature, where it does not present neither hemicelluloses nor lignin like plant cellulose. Due to the small fiber diameter of BC, the overall material presents high surface area and high porosity (Chawla, Bajaj, Survase, \& Singhal, 2009). It also possesses a high thermal stability and biocompatibility (Wang, Huang, Sheng, Lai, \& Xi, 2015). Moreover, BC can also incorporate polymerizable monomers into its network, occupying its void volume and interacting with the BC fiber chains. Researchers exploit this property in order to change and/or improve the characteristics of BC such as its hydrophobicity, electrical conductivity, surface reactivity, mechanical and thermal resistance, among others (Shi et al., 2011). Recently, several studies have been employed into improving the mechanical, thermal and electrical properties of cellulose using Silica and polyvinyl alcohol (Poyraz, Tozluoğlu, Candan, \& Demir, 2017; Poyraz, Tozluoğlu, Candan, Demir, \& Yavuz, 2017; Tozluoğlu, Poyraz, Candan, Yavuz, \& Arslan, 2017). With the production of bio-based products and the improvement of the initial properties of cellulose, these works show the potential application of these new materials to be implemented in different industrial sectors.

Conductive bacterial cellulose/polyaniline (BC/PANi) blends have been receiving increasing attention from the scientific community not only for electronic applications but also for the development of novel electro-responsive drug delivery mechanisms. To improve the electrical

\footnotetext{
* Corresponding author.

E-mail address: ncordeiro@staff.uma.pt (N. Cordeiro).
} 
properties of BC/PANi blends, Wang et al. (2012) have investigated the influence of the $\mathrm{pH}$ media, temperature, reaction time and molar ratios of the chemical components in the reaction while Catedral et al. (2004) and Liu, Tzou, and Gregory (1994) evaluated different types of protonic acids. Furthermore, with the same purpose of improving the electrical conductivity of these membranes, the incorporation of other elements into the reaction, such as carbon nanotubes and magnetite particles have been employed (Jasim, Shi, Lin, \& Yang, 2017; Park, Cheng, Choi, Kim, \& Hyun, 2013).

However, despite these recent advances, the impact of the $\mathrm{BC}$ matrix and chemical polymerization type (in situ and ex situ) into the electrical, structural and surface properties have not been studied. Thus, the current works aims to employ different BC matrixes (drained, freezedried and regenerated), as well as different polymerization methods (in situ and ex situ polymerization) to improve the initial properties of BC. The modifications made onto the initial $\mathrm{BC}$ matrixes will be monitored through FTIR-ATR, SEM-EDX, XRD, AFM, swelling, contact angle, electrical conductivity and IGC.

\section{Materials and methods}

\subsection{BC production}

Gluconacetobacter sp. was statically cultivated in Hestrin and Schramm (HS) medium (previously autoclaved during $15 \mathrm{~min}$ at $121^{\circ} \mathrm{C}$ ) to meet the bacteria cellular requirements for cellulose production. After being incubated for 7 days at $30^{\circ} \mathrm{C}$, the membrane was removed and washed with $\mathrm{NaOH} 0.5 \mathrm{M}$, at $80^{\circ} \mathrm{C}$ during $2 \mathrm{~h}$, and then neutralized with distilled water. The membrane was stored at $5{ }^{\circ} \mathrm{C}$ until further use.

\subsection{BC matrix}

Different BC matrixes were used for the synthesis of the BC/PANi membranes, which comprised in the use of drained, freeze-dried and regenerated $\mathrm{BC}$. Oven dried $\mathrm{BC}$ (OD-BC) was obtained by placing a wet $\mathrm{BC}$ membrane on the oven at $40^{\circ} \mathrm{C}$. Drained $\mathrm{BC}$ was obtained through manual pressing, removing up to $90 \%$ of water, while freeze-dried $\mathrm{BC}$ (FD-BC) was obtained by freezing at $-20^{\circ} \mathrm{C}$ during $30 \mathrm{~min}$, followed by freeze-drying.

To obtain a regenerated BC (R-BC), a wet membrane was dried on the oven at $40{ }^{\circ} \mathrm{C}$, cut into small pieces and a certain amount of $\mathrm{BC}$ was added to a solution of $\mathrm{LiCl} 8 \%(\mathrm{w} / \mathrm{v})$ in dimethylacetamide, obtaining a BC concentration of $0.5 \%(\mathrm{w} / \mathrm{v})$. Then, the mixture was placed in an oil bath at $110^{\circ} \mathrm{C}$ during $1 \mathrm{~h}$ followed by an ultrasonic bath during $1 \mathrm{~h}$ at room temperature (Ultrasons- $\mathrm{H}$, Selecta). The mixture was left under moderate stirring overnight, obtaining a clear viscous solution.

The regeneration process was employed by adding water in the dissolved $\mathrm{BC}$ solution, leaving under slow stirring $(<100 \mathrm{rpm})$ during $1 \mathrm{~h}$ to gain some firmness. Afterwards, the samples were washed through dialysis during $72 \mathrm{~h}$ using a dialysis tubing (benzoylated) with a molecular weight cut-off of 2000 Da (Sigma Aldrich) and then oven dried at $40{ }^{\circ} \mathrm{C}$.

\subsection{PANi synthesis conditions}

All polymerization reactions occurred under $24 \mathrm{~h}$ and under low stirring $(<100 \mathrm{rpm})$. The membranes were washed thoroughly until no aggregates could be observed. Then, the resulting blends were oven dried at $40^{\circ} \mathrm{C}$. A BC:ANi mass ratio of 0.10 , as well as a Ani:HCl:APS molar ratio of 1:1.2:1 was employed, following the optimal conditions reported by Wang et al. (2012). The reactions occurred under an inert atmosphere $\left(\mathrm{N}_{2}\right)$ and both monomer and persulfate aqueous solutions were dissolved in the presence of $\mathrm{HCl}$ (Riedel-de Haen).

The determination of the polymer content in the membranes was determined through the Eq. (1), where $w_{\text {composite }}$ corresponds to the dried weight of the blend and $w_{B C}$ corresponds to the dry weight of $\mathrm{BC}$ used for the BC/PANi synthesis.

Polymer content $=\left(\mathrm{w}_{\text {blend }}-\mathrm{w}_{\mathrm{BC}} / \mathrm{w}_{\text {blend }}\right) \times 100$

\subsection{Preparation of the $B C / P A N i$ membranes}

Different methods were used to obtain different BCANi membranes, using different $\mathrm{BC}$ matrixes (drained, freeze-dried and regenerated) as well as the different polymerization methods (in situ and ex situ).

The first set of BC/PANi blends was obtained by using a BC membrane (either drained (D-) or freeze dried (FD-)), and letting stand during $1 \mathrm{~h}$ with an aniline solution in order to incorporate the monomer prior to the addition of the oxidizing agent (ammonium persulfate (APS, Sigma Aldrich)). This way, it is expected that the polymerization of aniline occurs inside of the membranes, being considered an in situ polymerization. This way, the BC/PANi blends obtained were named DIS and FD-IS, being the prefix the BC used (drained or freeze-dried) and the suffix (IS) the type of polymerization used (in situ).

Likewise, the second set of BC/PANi blends obtained comprised in the use of a $\mathrm{BC}$ membrane (drained or freeze-dried) but this time aniline and APS were added at the same time. This way it is expected that the polymerization reaction would occur outside of the membrane and thus, being considered $\boldsymbol{e x}$ situ polymerization. The second set of BC/ PANi blends nomenclature followed the same pattern, being named accordingly D-ES and FD-ES, where the prefix refers the type of BC used (drained or freeze-dried) and the suffix (IS) to the type of polymerization used (ex situ).

Then, the third set consists in the in situ polymerization of aniline in the presence of dissolved BC. Pure aniline was added into dissolved BC (see Section 2.2), letting under low stirring $(<100 \mathrm{rpm}$ ) during $4 \mathrm{~h}$ in order for full homogenization. Then, it was added of the oxidizing agent (APS), which not only made aniline polymerize but also made BC to regenerate. This way, a 2-in-1 step method was employed, regenerating $\mathrm{BC}$ at the same time of PANi synthesis. Following the same nomenclature as the other samples, this membrane was called R-IS, being the prefix (R-) a reference to the $\mathrm{BC}$ present (regenerated $\mathrm{BC}$ ) and the suffix (IS) a reference to the polymerization method employed (in situ).

\subsection{Fourier transformed infrared spectrometer coupled to attenuated total reflectance (FTIR-ATR)}

FTIR-ATR spectra of the samples were obtained with a Perkin Elmer Spectrum Two coupled with a Diamond ATR accessory (DurasamplIR II, Smiths Detection, UK). 32 scans were acquired in the range of $4000-650 \mathrm{~cm}^{-1}$, with a wavenumber resolution of $4 \mathrm{~cm}^{-1}$.

\subsection{Scanning electronic microscopy coupled with energy dispersive X-ray spectroscopy (SEM-EDX)}

The samples were mounted and gold-coated in preparation for the SEM-EDX imaging analysis, performed using a scanning electron microscope SU3500. SEM images were obtained using a magnification of $3000 \times$. The EDX analysis was performed under an accelerated voltage of $5 \mathrm{kV}$, with the aim to identify the chemical compositions of samples at the surface, determining the weight percentages (wt.\%) of elements C, O, N, S and Cl.

\section{7. $X$-ray diffraction $(X R D)$}

XRD measurements were carried out with a Phillips X'pert MPD diffractometer using $\mathrm{Cu} \mathrm{K \alpha}$ radiation ( $\lambda$ of $1.54 \AA$ ) operating at $45 \mathrm{kV}$ and $40 \mathrm{~mA}$. The $2 \theta$ range under analysis was of $5-60^{\circ}$. The crystallinity index $(C I)$ of the samples was determined through Segal, Creely, Martin, and Conrad (1959) method, according to the eq. (2) where $I_{a m}$ corresponds to the intensities of the amorphous region $\left(18.0^{\circ}\right.$ and $13.8^{\circ}$ for type I and type II cellulose, respectively) and $I_{110}$ correspond to the 
intensity at the (110) plane $\left(22.7^{\circ}\right.$ and $21.0^{\circ}$ for type I and type II cellulose, respectively).

$C I=\left(1-I_{a m} / I_{110}\right) \times 100$

\subsection{Atomic force microscopy (AFM)}

To evaluate the surface topography of the samples, the AFM analysis was employed by an atomic force microscope (AFM, Solver PRO, NTMDT, Russia) in tapping mode in air atmosphere. Samples were scanned with the standard $\mathrm{Si}$ (silicon) cantilever with a force constant of $22 \mathrm{~N} / \mathrm{m}$ and at a resonance frequency of $325 \mathrm{kHz}$ (tip radius was $10 \mathrm{~nm}$ and the tip length was $95 \mu \mathrm{m}$ ), using a scan rate of $1.3 \mathrm{~Hz}$. The surface roughness $\left(R_{q}\right)$ was measured from representative images at $5 \times 5 \mu \mathrm{m}^{2}$ area, which corresponds to the average value of the surface height.

\subsection{Swelling capacity}

Swelling studies were performed according to Figueiredo et al. (2013) in order to evaluate the rehydration ability of the obtained membranes. This procedure comprises in the monitorization of the membrane's weight increase in distilled water, at room temperature, during $48 \mathrm{~h}$. The samples were removed from the water and their surfaces were carefully wiped with dry filter paper for weight purposes. The swelling ratio $(S W)$ was then determined from Eq. (3), where $W_{s}$ and $W_{d}$ are the weight of the swollen and dried membrane respectively.

$S W(\%)=\left(\mathrm{W}_{\mathrm{s}}-\mathrm{W}_{\mathrm{d}} / \mathrm{W}_{\mathrm{d}}\right) \times 100$

\subsection{Contact angle measurement}

A water droplet was aliquoted onto each membrane and the contact angle measurement was recorded with Krüss DSA-100 contact angle analyser at, by using on average $10 \mu \mathrm{L}$ of ultrapure water. The image was processed with the software imageJ using the plug-in drop_analysis, using the low-bond axisymmetric drop shape analysis approach ((LB-ASDA)) developed by Stalder, Kulik, Sage, Barbieri, and Hoffmann (2006).

\subsection{Electrical conductivity measurement}

The BC/PANi blend conductivity measurements were made at room temperature using the 4-probe technique (Panta \& Subedi, 2012). The system was comprised by a Fluke 87 RMS multimeter (to measure the current), a Leader multimeter $856 \mathrm{G}$ (to measure the voltage) and a CV regulated power supply Lab 502. Since the sample thickness $(t, \mu \mathrm{m})$ is lower than the probe spacing $(\mathrm{S}, \mu \mathrm{m})$, the conductivity $(\sigma, \mathrm{S} / \mathrm{cm})$ was measured according to the Eq. (4) where $V$ is the voltage and I (A) the current intensity.

$\sigma=(\ln (2) / \pi t) \times(I / V)$

\subsection{Inverse gas chromatography (IGC)}

IGC measurements were carried out on a commercial inverse gas chromatograph (Surface Measure Systems London, UK), equipped with both FID and TCD detector. The system was automatized with the Software SMS IGC Controller v1.8. Silanized glass columns with $3 \mathrm{~mm}$ inner diameter and $300 \mathrm{~mm}$ length were used (dimethyldichlorosilane, Repelcote $\mathrm{BDH}, \mathrm{UK})$, packing the sample through vertical tapping. The samples were conditioned overnight followed by $2 \mathrm{~h}$ at temperature measurement to stabilize.

Dispersive surface energy measurements were carried using a series of $n$-alkanes, from octane to undecane. For specific surface energy measurements, tetrahydrofuran, dichloromethane, ethyl acetate, acetonitrile and ethanol were used. In all current analyses, a concentration of $0.2 \mathrm{p} / \mathrm{p}_{0}$ was employed as well carried with a flow rate of $10 \mathrm{~mL} / \mathrm{min}$ at $25^{\circ} \mathrm{C}$ for the BC matrixes, $40-55^{\circ} \mathrm{C}$ for the BC/PANi blends and at $70-85^{\circ} \mathrm{C}$ for PANi sample. The higher temperatures applied for the blends and PANi powder is due to the strong interactions of the probes with the samples.

The probes were supplied by Sigma Aldrich, with analytic grade (>99\%). Methane was used as an inert reference gas and Helium was used as the carrier gas, both supplied by Air Liquide Company, with a purity above $99 \%$. The physical constants used in IGC and the equations used are described in Cordeiro, Gouveia, Moraes, and Amico (2011).

\subsection{Statistical analysis}

The statistical analysis of the data was carried using the IBM SPSS Statistics 23 software. Differences in the measurements of a given parameter were assessed by one-way analysis of variance (ANOVA), followed by a Tukey's post hoc analysis. For IGC, the error of the measurements was of $3 \%$ and as such the upper and lower values from the experimental value were determined. $p$-values of $<0.05$ were considered statistically different.

\section{Results and discussion}

3.1. Fourier transformed infrared spectrometer coupled to attenuated total reflectance (FTIR-ATR)

FTIR-ATR was employed to study the influence of the BC matrix processing methods as well the polymerization effect onto the functional groups onto BC. In Fig. 1A, it is possible to observe that all BC samples have the characteristic peaks of cellulose membranes: the peaks obtained at 1632 and $666 \mathrm{~cm}^{-1}$, correspond to the O-H bending and out of plane bending respectively; the peaks obtained at 1434, 1373, 1312 and $1051 \mathrm{~cm}^{-1}$ correspond to the $\mathrm{CH}$ and $\mathrm{CH}_{2}$ bond
A

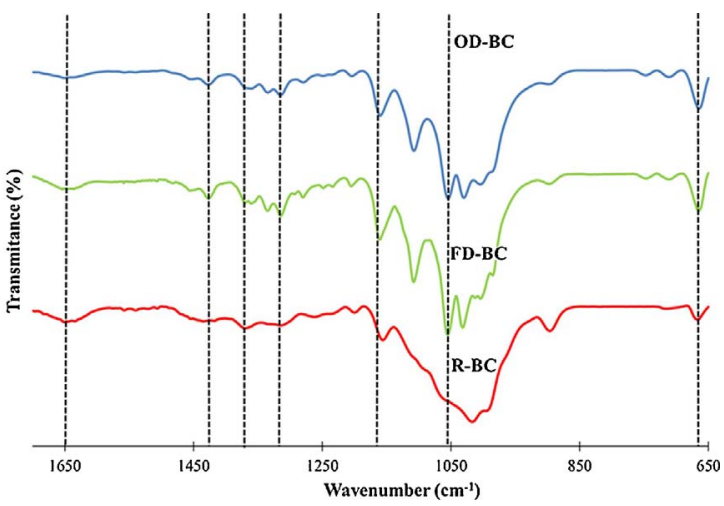

B

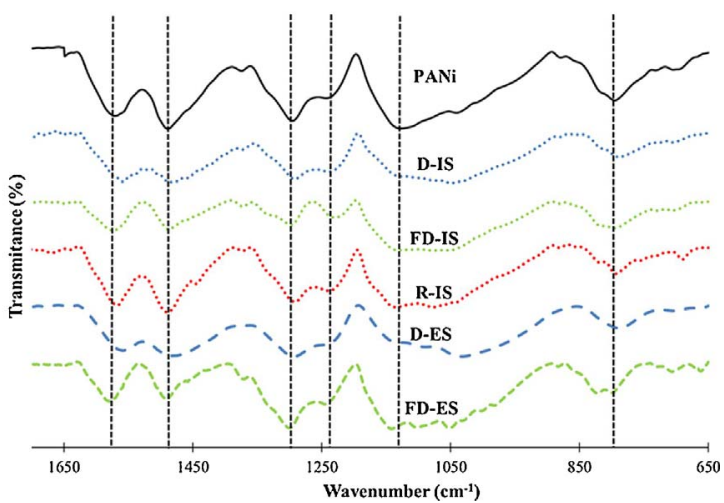

Fig. 1. FTIR-ATR spectra of the (A) BC matrixes and (B) PANi polymer and BC/PANi blends. 


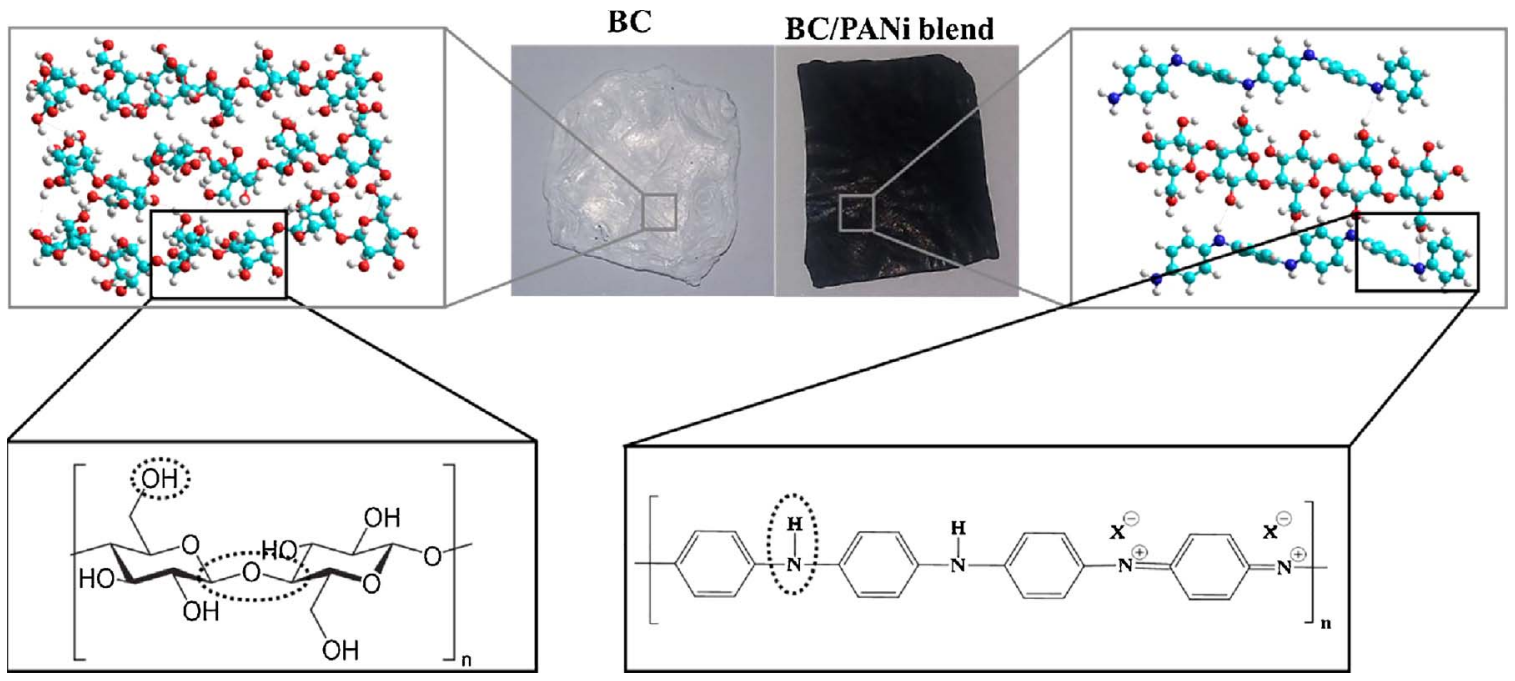

Fig. 2. Schematic representation of the PANi interaction with BC and respective functional groups.

vibrations and the peak at $1162 \mathrm{~cm}^{-1}$ corresponds to the stretching of ether groups (C-O-C), which can be found within the glucopyranose ring and between the glucose monomers (Fan, Dai, \& Huang, 2012). FTIR-ATR suggests that no changes occurred in the BC matrix functional groups composition through the employment of the different processing methods. However, the FTIR-ATR of R-BC predicted changes in the morphology of the cellulosic matrix.

The polymerization of aniline onto BC network was confirmed through the comparison of both PANi and BC/PANi blends spectra (Fig. 1B) with the respective BC matrixes. PANi presents characteristic peaks at 1557 and $1128 \mathrm{~cm}^{-1}$, corresponding to the stretching of the $\mathrm{C}-\mathrm{N}$ of benzenoid ring and peaks at $1483 \mathrm{~cm}^{-1}$, which correspond to the stretching of $\mathrm{C}=\mathrm{N}$ of the quinoid ring (Zhang, Zhang, Wang, \& Piao, 2013). Also, the peaks at 1290 and $1235 \mathrm{~cm}^{-1}$ correspond to the stretching vibrations of $\mathrm{C}-\mathrm{N}$ from secondary aromatic amines and $\mathrm{C}-\mathrm{N} .^{+}$in the polaron lattice of PANi, respectively (Trchová \& Stejskal, 2011). The peak at $788 \mathrm{~cm}^{-1}$ is related to the 1,4-disubstitution of the aromatic ring (Langer, 1990), which agrees with the chemical structure of PANi (Fig. 2). All the blends spectra present these characteristic peaks. Thus, FTIR-ATR indicates that both in situ and ex situ polymerization methods incorporated PANi in the different BC matrixes, resulting in the successful synthesis of BC/PANi blends.

\subsection{Electron dispersive $X$-ray spectroscopy (EDX)}

EDX was employed to evaluate the elemental composition at the samples surface. The $\mathrm{BC}$ matrixes, prior to modification, presented only the elements $\mathrm{C}$ and $\mathrm{O}$ (Table 1). The proportions of these two elements in the different $\mathrm{BC}$ matrixes indicates that it occurred changes in the surface group orientation with the use of different processing methods

Table 1

Elemental composition of the BC matrixes and BC/PANi blends.

\begin{tabular}{llllll}
\hline \multirow{2}{*}{ Sample } & \multicolumn{3}{l}{ Elemental composition (\%) } & & \\
\cline { 2 - 6 } & $\mathrm{C}$ & $\mathrm{O}$ & $\mathrm{N}$ & $\mathrm{S}$ & $\mathrm{Cl}$ \\
\hline OD-BC & 52.0 & 48.0 & n.d. & n.d. & n.d. \\
FD-BC & 59.4 & 40.6 & n.d. & n.d. & n.d. \\
R-BC & 48.0 & 52.0 & n.d. & n.d. & n.d. \\
D-IS & 71.1 & 14.2 & 10.7 & 4.0 & n.d. \\
FD-IS & 70.8 & 16.9 & 10.9 & 1.4 & n.d. \\
R-IS & 69.5 & 12.9 & 8.9 & 3.5 & 5.3 \\
D-ES & 68.5 & 14.8 & 7.9 & 8.1 & 0.7 \\
FD-ES & 73.2 & 12.0 & 12.3 & 2.2 & 0.3 \\
\hline
\end{tabular}

onto BC.

With the incorporation of PANi, the surface evidences the presence of $\mathrm{N}$, along with an increase in the $\mathrm{C}$ due to the aromatic ring of PANi and a consequent decrease on the amount of O. Different amounts of C, $\mathrm{N}$ and $\mathrm{O}$ in the BC/PANi blends predict differences in PANi incorporation into BC. Moreover, the element $\mathrm{S}$ can be found on the blends due to the persulfate used during the PANi synthesis, which is converted into sulphate and might establish ionic interactions with the amine groups (Shi et al., 2011). The presence of $\mathrm{Cl}$ in R-IS (5.3\%) can indicate that $\mathrm{Cl}$ is retained in the cellulose chains. This way, EDX further corroborates with FTIR-ATR that the BC/PANi blends were successfully synthesized and show that the $\mathrm{BC}$ matrix and polymerization methods affect the surface elemental composition, derived from the different PANi arrangements.

\subsection{X-ray dispersive spectroscopy (XRD)}

The morphological changes on BC with the different processing methods as well as with PANi incorporation were studied through XRD. Observing Fig. 3A, both OD-BC and FD-BC present a XRD profile of cellulose type I, with characteristic peaks found at $14.5^{\circ}, 16.73^{\circ}$ and $22.7^{\circ}$, corresponding to the (100), (010) and (110) planes, respectively (Poletto, Pistor, \& Zattera, 2013; Yudianti et al., 2016). In case of R-BC, the peaks corresponding to the (110) and (002) are now found at $11.9^{\circ}$ and $21.0^{\circ}$ respectively (Lima, Dierakowski, Tischer, \& Tischer, 2009; Liu et al., 2011; Zhang et al., 2016). The peak broadening on XRD also indicates a lower crystallite size $(1.14 \mathrm{~nm})$, in comparison to both OD$\mathrm{BC}$ and FD-BC (4.80 and $4.73 \mathrm{~nm}$ respectively) due to the amorphous content interference (Zugenmaier, 2008).

OD-BC presented a CI of $79.2 \%$, similar to FD-BC, indicating that the same BC crystallinity is obtained by using both of drying methods. These crystallinity values are comparable to previous studies on $\mathrm{BC}$ (Mohammadkazemi, Faria, \& Cordeiro, 2016; Tsouko et al., 2015; Yudianti et al., 2016) . However, R-BC presents a significant lower CI (39.8\%) which can be attributed to the rearrangement of the BC chains during the regeneration process, leading into a higher amorphous content (Liu et al., 2011). Thus, XRD indicates that the BC matrix processing methods affect the crystallinity of the resulting BC.

With PANi incorporation into BC lower crystallinity indexes were obtained due to the presence of PANi which breaks the bonds between BC chains, as seen in Fig. 3B. Minor differences in the BC crystallinity of the blends are observed upon the different polymerization methods used (up to $14 \%$ ). On the other hand, the crystallinity was greatly influenced by the BC matrix used on the polymerization process (Fig. 3B). The crystallinity of $\mathrm{BC}$ in the drained $\mathrm{BC}$ blends greatly reduces (about 
A

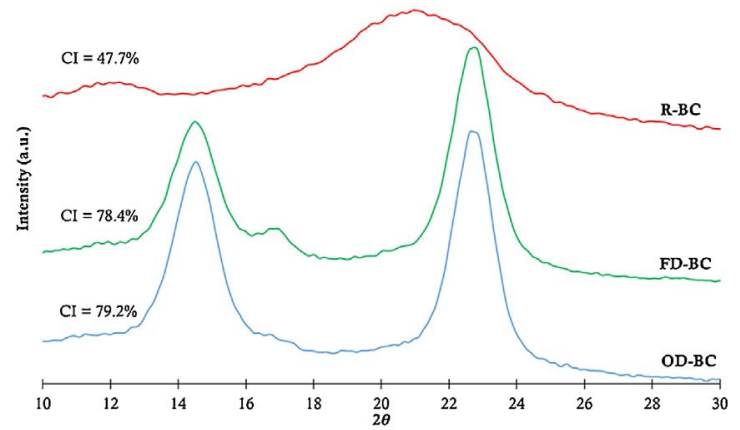

B

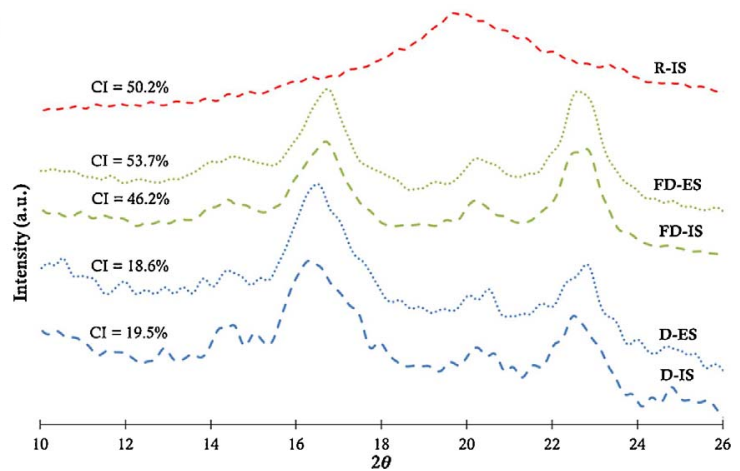

Fig. 3. XRD profile and crystallinity indexes of the (A) BC matrixes and (B) BC/PANi blends.

$60 \%$ ), whereas for the freeze-dried BC blends it decreases moderately (around 30\%) in comparison to the corresponding BC matrix. For R-IS, the $\mathrm{BC}$ crystallinity is similar to R-BC. This way, XRD shows that the BC matrix have a greater influence in the crystallinity of the blend when compared to the polymerization process.

\subsection{Scanning electronic microscopy (SEM) and atomic force microscopy} (AFM)

SEM and AFM were carried out in the current study with the aim to observe the influence of the $\mathrm{BC}$ matrix processing methods as well as the PANi incorporation into the morphology of the $\mathrm{BC}$ matrixes. OD-BC presents entangled fibrils (Fig. 4), which is further evidenced in FD-BC, due to the preservation of its $3 \mathrm{D}$ structure. Also, it can be observed that OD-BC is less porous than FD-BC. This can be due to the collapse of the pores during oven drying. In case of R-BC, a smooth and compact surface is observed, with an absence of fibrils at the surface. The compact surface of R-BC is formed by the regeneration process, with a fast chain aggregation which unables the creation of an organized structure. Therefore, an amorphous and compact BC is formed. This way, SEM shows that the different $\mathrm{BC}$ matrix processing methods greatly influence the BC matrix morphology.

The presence of PANi can be seen at the surface by the SEM micrographs (Fig. 4). Both drained BC blends (D-IS and D-ES) present fibers with a higher width when compared to OD-BC. In case of both freeze-dried BC blends (FD-IS and FD-ES), the incorporation of PANi is slightly different, being observed flakes/granules, and nearly absence of fibers at the surface. On R-IS, the surface became much rougher. Furthermore, in all blends, a more compact surface is observed, indicating the pore obstruction of the BC matrixes by PANi incorporation. Thus, the SEM micrographs shows changes in the PANi incorporation, according to the $\mathrm{BC}$ matrix employed.

The surface morphology was further explored by AFM where smooth surfaces for the BC matrixes are observed. Differences in the $R_{q}$ values are obtained (Fig. 5), which further corroborates that the $\mathrm{BC}$ matrix processing methods influenced in the morphology of $\mathrm{BC}$, as seen by SEM. With PANi incorporation, an increase in the surface roughness is observed. No significant differences are observed between in situ and ex situ polymerization (up to 4\%). However, when using different BC matrixes, different surface roughness values are obtained. It is seen that using drained $\mathrm{BC}$ results in a rougher $\mathrm{BC} / \mathrm{PANi}$ blend in comparison to freeze-dried BC (up to 14.4\%). In case of R-IS, it presented the greatest increase in the surface roughness in comparison to the remaining blends (68.5\%). Likewise, the AFM results suggests that the PANi incorporation is influenced by the BC matrix used.

\subsection{Swelling and contact angle analysis}

It is known that the hydrophilic nature and water retention capacity of $\mathrm{BC}$ is positively affected by the fibril arrangement and high surface area per unit mass (U1-Islam, Khan, \& Park, 2012). Hence, swelling studies were performed to study the effect of the $\mathrm{BC}$ matrix processing methods as well as the effect of the $\mathrm{BC}$ matrix and polymerization methods onto the water retention capacity of the different blends. ODBC presents a $S W_{\max }$ of $504 \%$ (Fig. 6) while for FD-BC, the rehydration ability is far superior (up to 9 times). This can be due to the BC pore preservation during the freeze-drying process. In case of the R-BC, a much lower $S W_{\max }$ was observed (50\%), evidencing the reduced reabsorption ability due to the compact structure. Thus, the different processing techniques altered the BC pore availability and subsequently $S W_{\max }$, being the freeze-drying method the most suited method to obtain a highly porous $\mathrm{BC}$ membrane with a higher water retention.

With PANi incorporation, a significant decrease in the $S W_{\max }$ was observed (Fig. 6), which can be due to the obstruction of pores by PANi, as well as due to its hydrophobic nature. Taking the respective $\mathrm{BC}$ matrix as a reference, for FD-BC/PANi blends the swelling ratio decreases more significantly (around 90\%) when compared to the remaining blends (60\%). Moreover, both FD-IS and FD-ES blends presented a higher swelling ratio when compared to D-IS and D-ES blends, while R-IS presented the lowest swelling. This way, it can be concluded that the water reabsorption capacity of the BC/PANi blends is highly influenced by the BC matrix used.

The contact angle measurements were performed for the several BC matrixes to search for changes in their hydrophilicity (Fig. 7). OD-BC presented a contact angle of $47.6^{\circ}$, whereas by freeze-drying it is greatly reduced $\left(23.7^{\circ}\right)$, which indicates a higher hydrophilic behaviour. In contrast, R-BC presented a higher contact angle $\left(54.0^{\circ}\right)$, indicating an increase in the hydrophobic behaviour. These results corroborate the swelling studies where followed the same trend as well as indicated that by freeze-drying confers BC a more hydrophilic behaviour, whereas by regeneration a more hydrophobic $\mathrm{BC}$ is obtained.

Regarding the $\mathrm{BC} / \mathrm{PANi}$ blends, it was observed an increase in the contact angle when compared to the respective BC matrix (1.1-2.7 times higher). The D-IS blend presented the lowest contact angle $\left(54.3^{\circ}\right)$ whereas R-IS presented the highest contact angle $\left(85.6^{\circ}\right)$. However, the contact angle analysis was unable to discern any direct relation with the synthesis conditions employed.

\subsection{Polymer uptake and electrical conductivity}

The polymer uptake and electrical conductivity of BC/PANi blends were evaluated, being found in Fig. 8. Regarding the different BC matrixes used, both D-IS and D-ES blends presented lower polymer contents in comparison to FD-IS and FD-ES respectively, which indicates the influence of the $\mathrm{BC}$ matrix used. As seen above, FD-BC matrix presents a high porosity and high swelling (Fig. 6), which contributes to higher incorporation yields when compared to drained BC. On the other hand, the electrical conductivity of the drained BC/PANi blends is higher when compared to the remaining blends. These differences could indicate changes in the PANi chain orientation and/or molecular weight with the use of different $\mathrm{BC}$ matrixes. More specifically, it means that FD-BC network is unfavorable for PANi chain growth, leading to a 

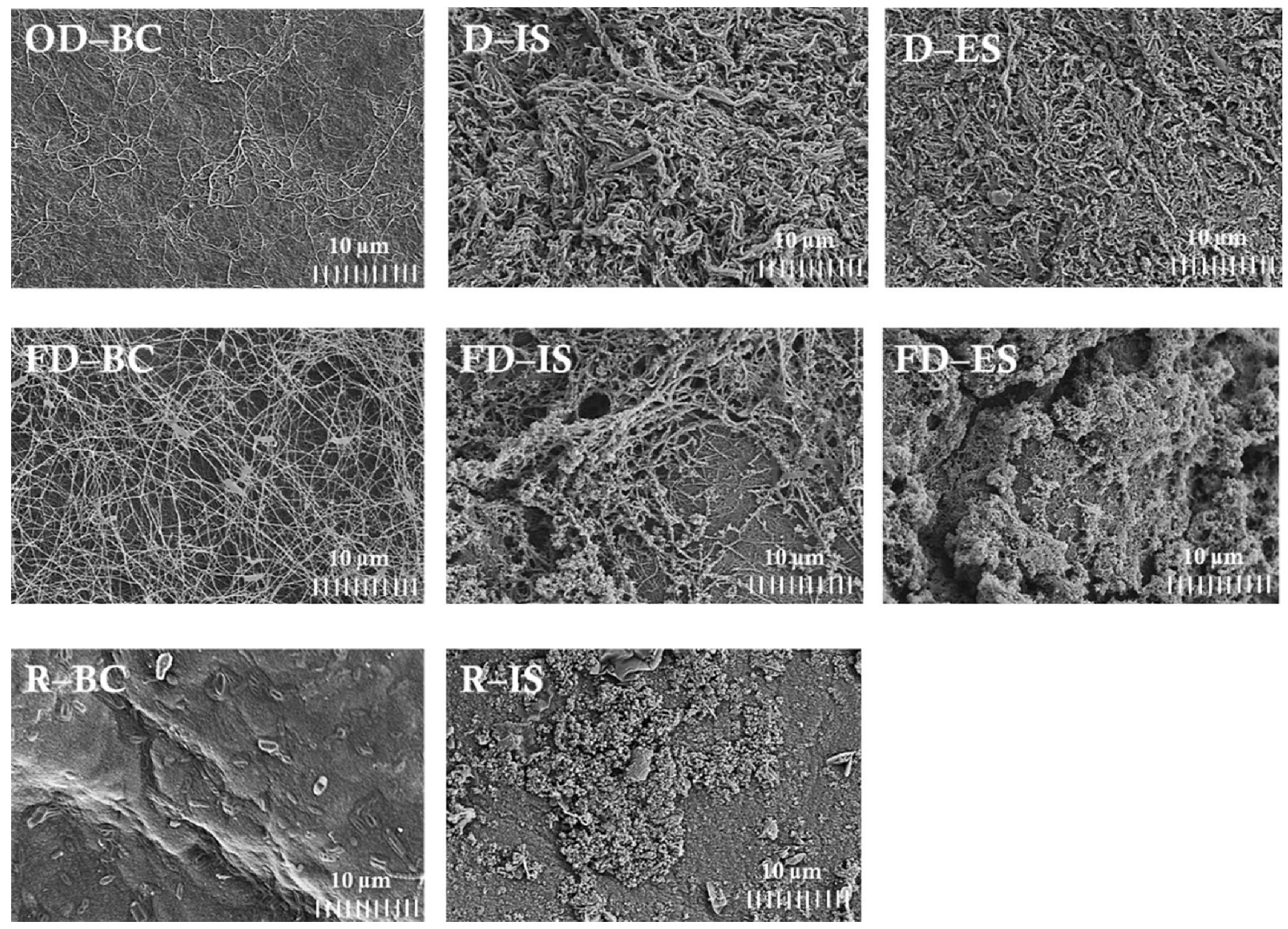

Fig. 4. SEM micrographs $(3000 \times)$ of $B C$ matrixes and BC/PANi blends.

lower ability to conduct electricity.

Regarding the synthesis method, in situ polymerization positively influenced the polymer content incorporated as well as the electrical conductivity (1.9-2.6 times higher) than the blends obtained through ex situ chemical polymerization. All of the BC/PANi blends obtained fall within the range of semiconductive materials, which ranges $10^{-7}-10^{\circ}$ S/cm (Kaur et al., 2015).

Through the polymer uptake and electrical conductivity analysis it is possible to observe that in situ polymerization promotes higher PANi contents as well as higher conductivities. Moreover, the use of a drained BC membrane over a freeze-dried or regenerated lead to higher conductivities. Thus, the use of drained BC via in situ polymerization should be highlighted as the most suitable method for highly conductive $\mathrm{BC}$ / PANi blends.

\subsection{Surface properties}

IGC analysis has been used to observe changes in the surface properties with the different $\mathrm{BC}$ matrix processing methods, as well as to observe any physico-chemical changes that occurred during the synthesis of the blends. This way, it would provide further information regarding the influence of the $\mathrm{BC}$ matrix and synthesis method in the final properties of the BC/PANi blends.

\subsubsection{Surface energy}

The dispersive surface energies $\left(\gamma_{s}^{d}\right)$ of the different BC matrixes obtained ranged between $35.6-37.6 \mathrm{~mJ} / \mathrm{m}^{2}$ (Table 2), which is close to values found the literature to BC, (Castro et al., 2015; Ferguson et al., 2016). The differences in the $\gamma_{s}^{d}$ values, indicates that the different $\mathrm{BC}$ matrix processing methods affected the orientation of the BC surface groups, as observed by EDX.

With PANi incorporation, the $\gamma_{s}^{D}$ of the BC matrixes significantly increases due to the PANi incorporation, which presents a high $\gamma_{s}^{D}$ value. Also, the increased $\gamma_{S}^{D}$ is corroborated by an increase of the $\mathrm{C}$ amount through EDX (Table 1), due to the $\mathrm{C}-\mathrm{H}$ groups of PANi aromatic ring. Higher $\mathrm{C}$ amounts are attained in FD-ES (highest $\gamma_{s}^{D}$ ) while lower $\mathrm{C}$ amounts were obtained for R-IS (lower $\gamma_{s}^{D}$ ). Moreover, the increased $\gamma_{s}^{d}$ can be related to an increase of the surface hydrophobicity, as seen through the contact angles.

Through the surface energy analysis, it was possible to obtain indication of changes in the surface group orientation during the different BC matrix processing methods as well it was obtained compelling data that suggests the PANi incorporation onto the membranes.

\subsubsection{Acid-base surface character}

Observing cellulose chemical structure, it is expected an amphoteric behaviour, with a predominantly acidic character, due to the electron acceptor (H from hydroxyl groups) and electron donor (O from glucosidic bonds and hydroxyl groups) groups (Fig. 2). OD-BC presented a $K_{b} / K_{a}$ of 0.7, which shows an acidic behaviour. Castro et al., 2015 and (Pommet et al., 2008) reported the same acidic behaviour as the obtained OD-BC in the current work (Table 2). In both FD-BC and R-BC the acidic behaviour is more pronounced $\left(K_{b} / K_{a}\right.$ of 0.6$)$, meaning that there is a higher number of acidic groups available in the surface, when compared to the basic groups. Thus, the $K_{b} / K_{a}$ values further indicates changes in the orientation of the surface groups with the different BC matrix processing methods.

For PANi a basic character is observed (Table 2), which results from the presence of amine groups throughout the polymer chains. With PANi incorporation in the $\mathrm{BC}$ matrixes, the $\mathrm{BC}$ acidic behaviour $\left(K_{b} / K_{a}\right.$ ranging from 0.6 to 0.8$)$ is shifted into a basic behaviour $\left(K_{b} / K_{a}\right.$ ranging from 1.9 to 3.8), corroborating the fact that the PANi polymer can be found at the surface. Also, the percentage of $\mathrm{N}$ and $\mathrm{O}$, presented in 


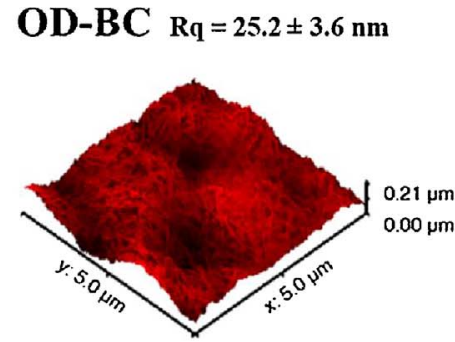

FD-BC $R q=16.2 \pm 2.7 \mathrm{~nm}$

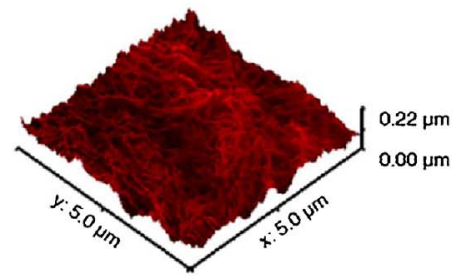

R-BC $R q=22.0 \pm 3.3 \mathrm{~nm}$

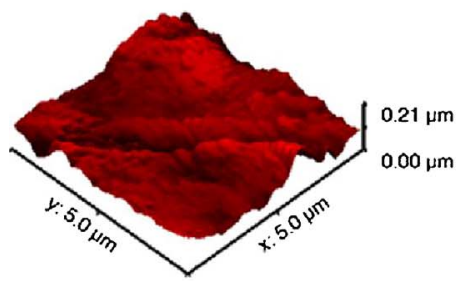

D-IS $\mathrm{Rq}=40.8 \pm 7.0 \mathrm{~nm}$

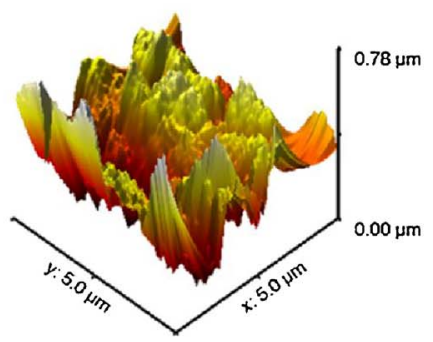

FD-IS $\mathrm{Rq}=36.5 \pm 2.9 \mathrm{~nm}$

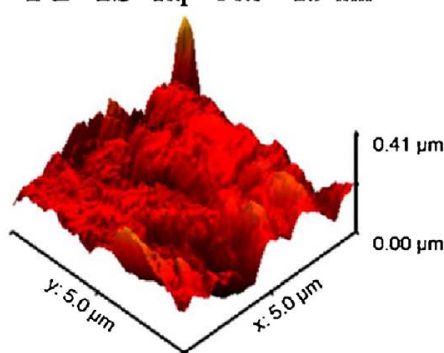

R-IS $\mathrm{Rq}=69.8 \pm 6.5 \mathrm{~nm}$

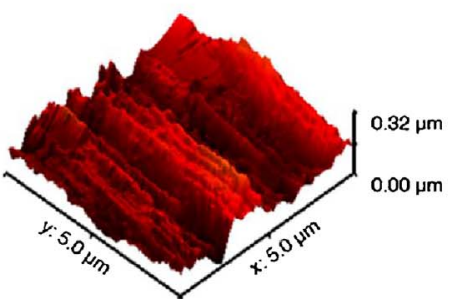

D-ES $\mathrm{Rq}=39.8 \pm 4.1 \mathrm{~nm}$

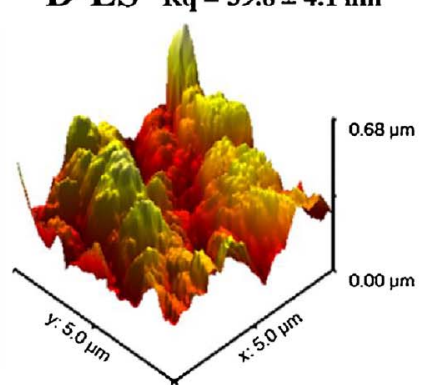

FD-ES $\mathrm{Rq}=34.8 \pm 3.8 \mathrm{~nm}$

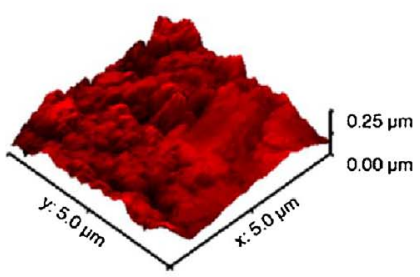

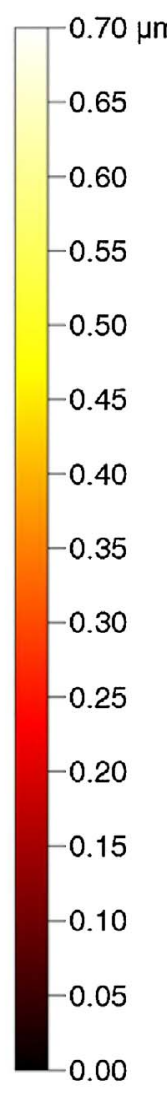

Fig. 5. 3D-AFM and surface roughness $\left(R_{q}\right)$ of BC matrixes and BC/PANi blends.

A

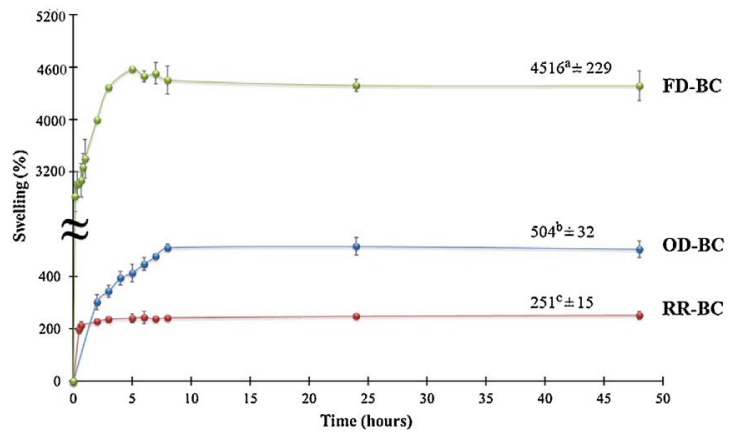

B

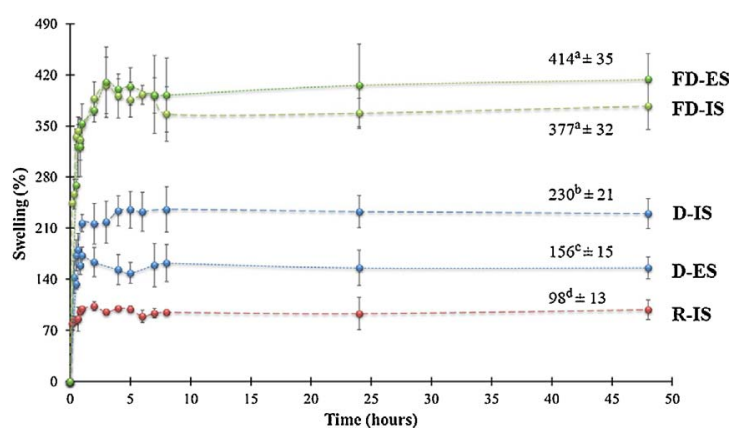

Fig. 6. Swelling behaviour of the (A) BC matrixes and (B) BC/PANi blends.

Table 1, gives an indication of the relative basicity of the BC/PANi blends. The percentage of $\mathrm{N}$ given by EDX (Table 1 ) is associated with the amine groups of PANi whereas the percentage of $\mathrm{O}$ is associated to the hydroxyl and ether groups of cellulose. When compared to the $K_{b} /$
$K_{a}$ values (Table 2), it is seen that the presence of higher $\mathrm{N}$ and lower $\mathrm{O}$ values are associated with higher $K_{b} / K_{a}$ values. This way, the acid base study through IGC can suggest changes in the PANi amount on the surface.

\section{Conclusion}

The current work aims to improve the $\mathrm{BC}$ properties through the employment of different $\mathrm{BC}$ matrixes as well as synthesis methods during the synthesis of the BC/PANi blends. The results demonstrated that the different processing methods applied onto the BC matrix greatly influence the morphology and the surface properties, which were reflected when used for the synthesis of the BC/PANi blends. The use of a drained $\mathrm{BC}$ membrane led into higher conductive $\mathrm{BC} / \mathrm{PANi}$ blends. Moreover, the use of different polymerization methods favors the use of in situ polymerization for the production of highly conductive membranes. Therefore, the current work evidence the influence of the different BC treatments, as well as the different polymerization methods, in the properties of the resulting blends. Furthermore, the obtained results encourage the use of this polymeric blend in further studies which could be applied in field of electronics due to its properties and rather ease in manufacture.

\section{Acknowledgments}

The authors would like to thank the Programa Nacional de Re-equipamento Científico, POCI 2010, for sponsoring IGC work (FEDER and Foundation for Science and Technology). Moreover, the help of Tomásia Fernandes and Igor Fernandes (Madeira University) in the laboratory work is appreciated. 

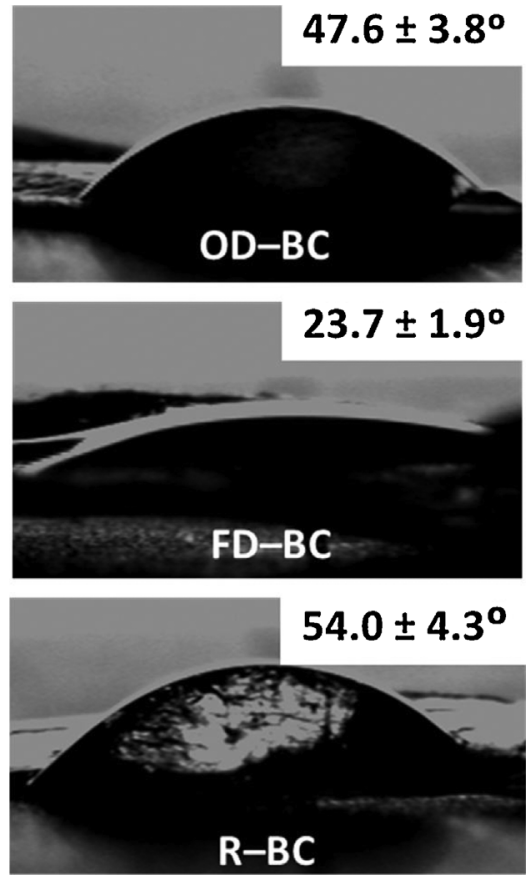

Fig. 7. Contact angle of the BC matrixes and BC/PANi blends.

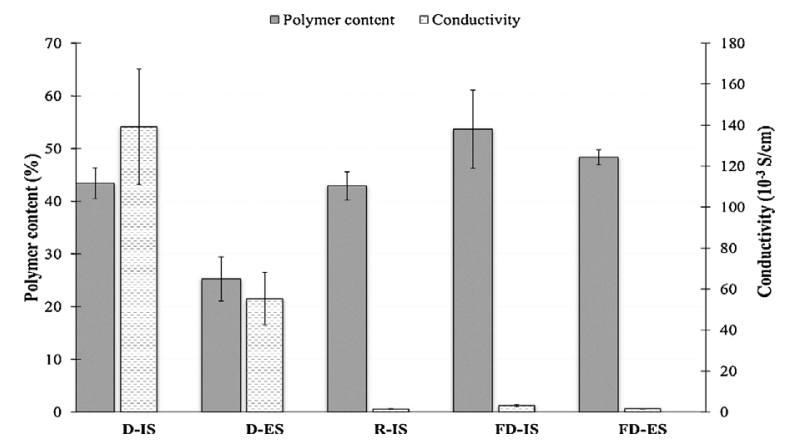

Fig. 8. Polymer content and electrical conductivity of the BC/PANi blends.

Table 2

Surface energy and acid/base behaviour of BC and respective blends at $25^{\circ} \mathrm{C}$.

\begin{tabular}{lll}
\hline Sample & $\gamma_{S}^{\mathrm{D}}\left(\mathrm{mJ} / \mathrm{m}^{2}\right)$ & $K_{b} / K_{a}$ \\
\hline OD-BC & $37.7^{\mathrm{a}} \pm 0.9$ & $0.8^{\mathrm{a}} \pm 0.0$ \\
FD-BC & $35.2^{\mathrm{ab}} \pm 0.9$ & $0.6^{\mathrm{b}} \pm 0.0$ \\
R-BC & $34.6^{\mathrm{b}} \pm 0.9$ & $0.6^{\mathrm{b}} \pm 0.0$ \\
PANi & $119.9^{\mathrm{a}} \pm 3.6$ & $3.5^{\mathrm{a}} \pm 0.1$ \\
D-IS & $64.4^{\mathrm{b}} \pm 1.9$ & $2.6^{\mathrm{b}} \pm 0.1$ \\
FD-IS & $67.7^{\mathrm{b}} \pm 2.0$ & $1.9^{\mathrm{c}} \pm 0.1$ \\
R-IS & $51.9^{\mathrm{c}} \pm 1.1$ & $1.9^{\mathrm{c}} \pm 0.0$ \\
D-ES & $63.7^{\mathrm{b}} \pm 1.9$ & $2.3^{\mathrm{d}} \pm 0.1$ \\
FD-ES & $75.0^{\mathrm{d}} \pm 2.3$ & $3.8^{\mathrm{e}} \pm 0.1$ \\
\hline
\end{tabular}

$\gamma_{s}^{d}$ - Dispersive component of the surface energy; $K_{b} / K_{a}$ - basicity ratio. Values in same column not sharing a common superscript are statistically different $(p<0.05)$.

\section{References}

Castro, C., Cordeiro, N., Faria, M., Zuluaga, R., Putaux, J., Filpponen, I., et al. (2015). Insitu glyoxalization during biosynthesis of bacterial cellulose. Carbohydrate Polymers, $126,32-39$.

Catedral, M., Tapia, A., \& Sarmago, R. (2004). Effect of dopant ions on the electrical condutivity and microstructure of polyaniline (emeraldine salt). Science Diliman, 16, 41-46.

Chawla, P., Bajaj, I., Survase, A., \& Singhal, R. (2009). Microbial cellulose: Fermentative production and applications. Food Technology and Biotechnology, 47, 107-124.

Cordeiro, N., Gouveia, C., Moraes, A., \& Amico, S. (2011). Natural fibers characterization by inverse gas chromatography. Carbohydrate Polymers, 84, 110-117.

Fan, M., Dai, D., \& Huang, B. (2012). Fourier transform infrared spectroscopy for natural fibres. In S. Mohammed Salih (Ed.). Fourier transform - Materials analysis (pp. 45-68). InTech.

Ferguson, A., Khan, U., Walsh, M., Lee, K., Bismarch, A., Shaffer, M., et al. (2016). Understanding the dispersion and assembly of bacterial cellulose in organic solvents. Biomacromolecules, 17, 1845-1853.

Figueiredo, A., Varona, A., Fernandes, S., Palomares, T., Azpeitia, E., Timmons, A., et al. (2013). Biocompatible bacterial Cellulose-poly(2-hydroxyethylmetacrylate) nanocomposite films. Biomed Research International, 2013, 1-14.

Jasim, A. U. M., Shi, Z., Lin, X., \& Yang, G. (2017). Fabrication of bacterial cellulose/ polyaniline/single-walled carbon nanotubes membrane for potential application as biosensor. Carbohydrate Polymers, 163, 62-69.

Kaur, G., Adhikari, R., Cass, P., Bown, M., \& Gunatillake, P. (2015). Electrically conductive polymers and composites for biomedical applications. Royal Society of Chemistry Advances, 5, 37553-37567.

Langer, J. (1990). N-substituted polyanilines II. Photoacoustic and FT-IR spectra of poly (N-methylaniline) and related copolymers. Synthetic Metals, 35, 301-305.

Lima, G., Dierakowski, M., Tischer, P., \& Tischer, C. (2009). Characterization of the bacterial cellulose dissolved on dimethylacetamide/lithium chloride. $10^{\circ}$ Congresso Brasileiro de polímeros. Foz de Iguaçu.

Liu, M., Tzou, K., \& Gregory, R. (1994). Influence of the doping conditions on the surface energies of conducting polymers. Synthetic Metals, 63, 67-71.

Liu, Z., Wang, H., Li, Z., Lu, X., Zhang, X., Zhang, S., et al. (2011). Characterization of the regenerated cellulose films in ionic liquids and rheological properties of the solutions. Materials Chemistry and Physics, 128, 220-227.

Mohammadkazemi, F., Faria, M., \& Cordeiro, N. (2016). In situ biosynthesis of bacterial nanocellulose- $\mathrm{CaCO}_{3}$ hybrid bionanocomposite: One-step process. Materials Science and Engineering: C, 65, 393-399.

Panta, G., \& Subedi, D. (2012). Electrical characterization of aluminium (Al) thin films measured by using four-point probe method. Kathmandu University Journal of Science, Engeneering and Technology, 8, 31-36.

Park, M., Cheng, J., Choi, J., Kim, J., \& Hyun, J. (2013). Electromagnetic nanocomposite of bacterial cellulose using magnetite nanoclusters and polyaniline. Colloids and Surfaces B: Biointerfaces, 12, 238-242.

Poletto, M., Pistor, V., \& Zattera, A. (2013). Structural characteristics and thermal properties of native cellulose. In G. L. Ven T (Ed.). Cellulose - Fundamental aspects. InTech.

Pommet, M., Juntaro, J., Heng, J., Mantalaris, A., Lee, A., Wilson, K., et al. (2008). Surface modification of natural fibers using bacteria: depositing bacterial cellulose onto natural fibers to create hierarchical fiber reinforced nanocomposites. Biomacromolecules, 9, 1643-1651.

Poyraz, B., Tozluoğlu, A., Candan, Z., Demir, A., \& Yavuz, M. (2017). Influence of PVA and silica on chemical, thermo-mechanical and electrical properties of celluclasttreated nanofibrillated cellulose composites. International Journal of Biological Macromolecules, 104, 384-392.

Sapurina, I., \& Shishov, M. (2012). Oxidative polymerization of aniline: Molecular synthesis of polyaniline and the formation of supramolecular structures. In A.d. S. Gomes (Ed.). New polymers for special applications. InTech.

Segal, L., Creely, J., Martin, A., \& Conrad, C. (1959). An empirical method for estimating the degree of crystallinity of native cellulose using the X-ray diffractometer. Textile 
Research Journal, 29, 786-794.

Shi, Z., Zang, S., Jiang, F., Huang, L., Lu, D., Ma, Y., et al. (2011). In situ nano-assembly of bacterial cellulose-polyaniline composites. RSC Advances, 2, 1040-1046.

Stalder, A., Kulik, G., Sage, D., Barbieri, L., \& Hoffmann, P. (2006). A snake-based approach to accurate determination of both contact points and contact angles. Colloids and Surfaces A, 286, 92-103.

Tozluoğlu, A., Poyraz, B., Candan, Z., Yavuz, M., \& Arslan, R. (2017). Biofilms from micro/nanocellulose of $\mathrm{NaBH}_{4}$-modified kraft pulp. Bulletin of Materials Science, 40, 699-710.

Trchová, M., \& Stejskal, J. (2011). Polyaniline: The infrared spectroscopy of conducting polymer nanotubes (IUPAC technical report). Pure and Applied Chemistry, 83, 1803-1817.

Tsouko, E., Kourmentza, C., Ladakis, D., Kopsahelis, N., Mandala, I., Papanikolaou, S., et al. (2015). Bacterial cellulose production from industrial waste and by-product streams. International Journal of Molecular Sciences, 16, 14832-14849.

Ul-Islam, M., Khan, T., \& Park, J. (2012). Water holding and release properties of bacterial cellulose obtained by in situ and ex situ modification. Carbohydrate Polymers, 88, 596-603.
Wang, H., Zhu, E., Yang, J., Zhou, P., Sun, D., \& Tang, W. (2012). Bacterial cellulose nanofiber-supported polyaniline nanocomposites with flake-shaped morphology as supercacitor electrodes. Journal of Physical Chemistry, 116, 13013-13019.

Wang, S., Huang, T., Sheng, L., Lai, C., \& Xi, T. (2015). Blood compatibility of oxidized bacterial cellulose/silk fibroin composite membrane. In S. L. Chung, \& X. Li (Eds.). Proceedings of 2014 international conference on materials science and energy engeneeringSingapore: World Scientific Publishing p. 700.

Yudianti, R., Syampurwadi, A., Onggo, H., Karina, M., Uyama, H., \& Azuma, J. (2016). Properties of bacterial cellulose transparent film regenerated from dimethylacetamide-LiCl solution. Polymers Advanced Technologies, 27, 1102-1107.

Zhang, D., Zhang, L., Wang, B., \& Piao, G. (2013). Nanocomposites of polyaniline and cellulose nanocrystals prepared in lyotropic chiral nematic liquid crystals. Journal of Materials, 1-6.

Zhang, H., Guo, H., Wang, B., Shi, S., Xiong, L., \& Chen, X. (2016). Synthesis and characterization of quaternized bacterial cellulose prepared in homogeneous aqueous solution. Carbohydrate Polymers, 136, 171-176.

Zugenmaier, P. (2008). Crystalline cellulose and derivatives: Characterization and structures (1st edition ed.). Springer. 